\title{
Successful Management of Pregnancy in Uncorrected Tetralogy of Fallot with Pulmonary Atresia
}

\author{
Agarwal Nutan · Gupta Monica • Singh Nilanchali • \\ Kachhawa Garima $\cdot$ Kriplani Alka
}

Received: 2 February 2015/Accepted: 16 February 2015/Published online: 26 March 2015

(C) Federation of Obstetric \& Gynecological Societies of India 2015

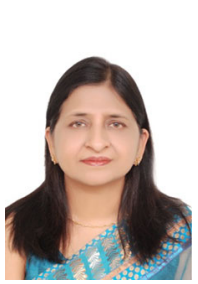

\section{About the Author}

Dr. Nutan Agarwal is the Editor-in-Chief of Indian Journal Obstetrics \& Gynaecology and the Assistant Editor of Asian Journal of Obstetrics \& Gynaecology practices. She is the Founder Secretary of Gynae-Endocrine Society of India (GESI) and the Chairperson of Gynae-Endocrine Society of AOGD. She is also the Organizing Secretary of the Endoscopy training program at the AIIMS. She has done Observership in fetal medicine unit at King College Hospital, London, UK and has Diploma in Obs-Gynae USG from Ian Donald Medical School University. She has 124 long papers and 195 abstracts published in various international and national Journals. She has been the principal investigator and co-investigator of 24 research projects and chief guide and Co-guide of 47 theses. She is the recipient of International Medical Excellence award for the health sector 2009 and G S Reddy Pondicherry oration award at the 7th International Congress on Geriatric Care.
\end{abstract}

\section{Introduction}

Tetralogy of Fallot (TOF) is the commonest congenital cyanotic heart disorder, accounting for 5-6\% of them [1]. It is characterized by a large ventricular septal defect, right ventricular outflow tract obstruction, right ventricular hypertrophy, and aorta overriding the interventricular septum. Most patients with TOF undergo reparative surgery in childhood. TOF with pulmonary atresia is the extreme end of anatomic spectrum which is difficult to correct surgically. Pregnancy in uncorrected TOF entails serious risks including increased maternal morbidity, mortality up to $15 \%$, and poor perinatal outcome [2]. We report a case of

Agarwal N. (凹), Professor · Gupta M., Senior Resident ·

Singh N., Senior Research Associate .

Kachhawa G., Assistant Professor · Kriplani A., Professor

Department of Obstetrics and Gynecology, All India Institute

of Medical Sciences, New Delhi, India

e-mail: nutan.agarwal1@gmail.com;

nutan_agarwal@yahoo.com pregnancy in uncorrected TOF with pulmonary atresia which was successfully managed at a tertiary care center with multidisciplinary approach.

\section{Case}

A 32-year old, $\mathrm{G}_{2} \mathrm{P}_{1} \mathrm{~L}_{0}$, was referred to our department at 33 weeks of pregnancy with uncorrected TOF with pulmonary atresia. At 3 months of age, she had failure to gain weight, feeding difficulty, and recurrent respiratory tract infection. She also had history of cyanosis and on-and-off dyspnea on heavy exertion and on evaluation was diagnosed with TOF at the age of 26 years. She was advised digoxin, torsemide, and sildenafil. Surgical repair of cardiac defect could not be done as the mediastinal pulmonary arteries were absent. She married at 27 years age, and after 3 years had a preterm vaginal delivery at 34 weeks, but the baby expired at 4 months after delivery due to unknown cause. Her antepartum and postpartum periods were uneventful with regard her cardiac status. 
At the time of presentation to our department, she was NYHA class II, and on examination had clubbing of all the fingers and toes (Fig. 1); cyanosis of lips and oral mucosa; fingers and toes with bilateral pitting pedal edema. Vitals were normal, and oxygen saturation was $82 \%$ at room air. She had precordial bulge, and, on auscultation, had pansystolic murmur. Uterine fundal height revealed growth lag of 3 weeks. Obstetrical investigations including fetal echo was normal. She had polycythemia with hemoglobin and hematocrit of $19 \mathrm{gm} / \mathrm{dl}$ and $58 \%$, respectively, and thrombocytopenia of $50,000 / \mu \mathrm{L}$. She also had hyperuricemia with uric acid levels of $10 \mathrm{mg} / \mathrm{dl}$. ECG revealed bifid $\mathrm{P}$ wave ( $\mathrm{P}$ pulmonale) and right ventrivular hypertrophy. Echo revealed large nonrestrictive VSD with right-to-left shunt, pulmonary atresia, absent mediastinal pulmonary arteries, multiple aortopulmonary collaterals, and no aortic arch coarctation with ejection fraction of $60 \%$. Previous chest x-ray showed a boot-shaped heart (Fig. 2). Ultrasound showed 4 weeks' growth lag in fetus and increased resistance in umbilical blood flow with a S/D ratio of 3.5.

After detailed cardiology consultation, metoprolol $50 \mathrm{mg}$ administration was started and labor induced with concentrated oxytocin at 37 weeks gestation in view of rising hematocrit of $63 \%$, worsening performance status, and fetal growth restriction. Within $8 \mathrm{~h}$ of induction, she delivered a $1.7 \mathrm{~kg}$, female baby vaginally. She had atonic postpartum hemorrhage of $900 \mathrm{ml}$ which was managed conservatively with oxytocics and bimanual massage. Arterial blood gas analysis revealed no acidosis and hemoglobin of $15 \mathrm{gm} / \mathrm{dl}$. Three hours after delivery, the patient had sudden attack of worsening cyanosis, desaturation $\left(\mathrm{O}_{2}\right.$ saturation $\left.<80 \%\right)$, and hypovolemic shock with unrecordable blood pressure, pulselessness, and cold extremities. Immediate transfusion of crystalloids, colloids,

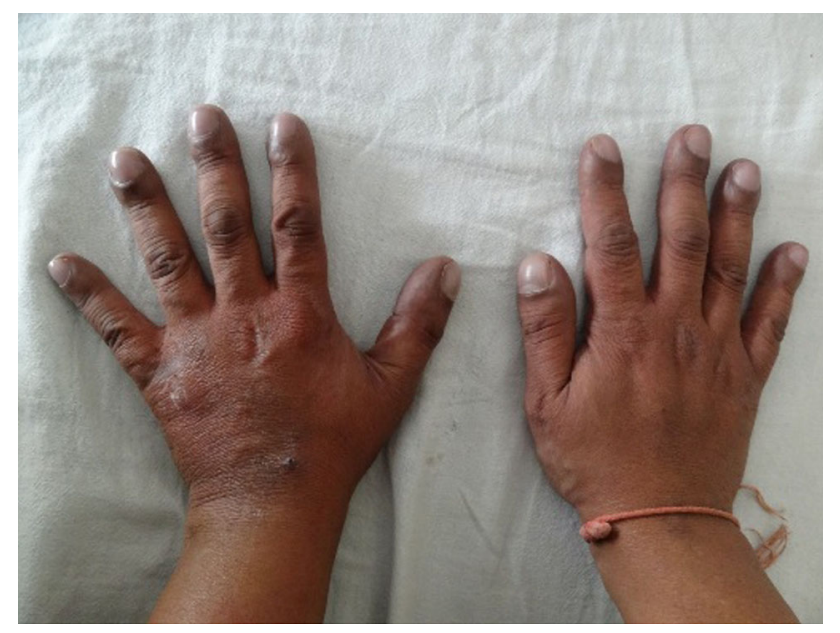

Fig. 1 Clubbing and cyanosis of finger nails

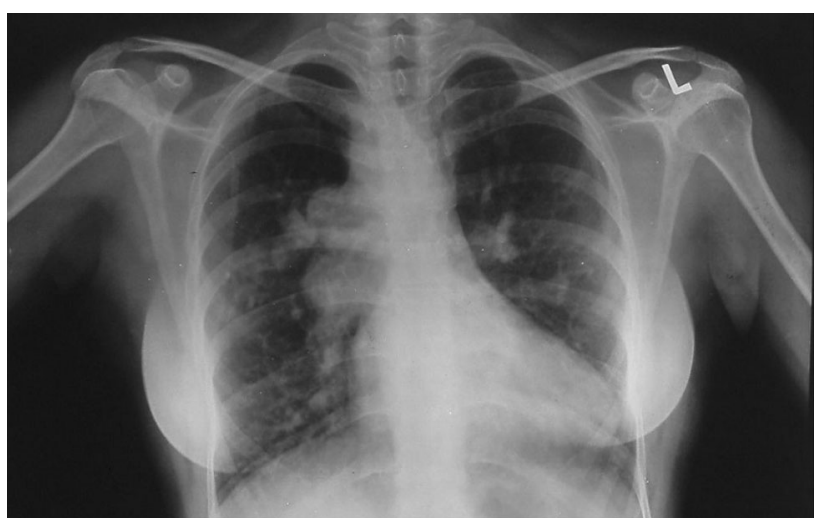

Fig. 2 Chest X-ray showing "boot-shaped heart"

two units of packed red blood cells was done, and cyanosis and hypotension managed with infusion of phenylephrine and dopamine. The patient was intubated as she was not able to maintain oxygen saturation despite all measures and shifted to intensive cardiac care unit. Next day, the patient improved, and she was extubated. However, she underwent some investigations and was found to have deranged liver and kidney function tests with hepatic transaminase up to $1500 \mathrm{IU} / \mathrm{L}$, serum urea of $70 \mathrm{mg} / \mathrm{dl}$, and creatinine $3.5 \mathrm{mg} / \mathrm{dl}$, suggestive of ischemic hepatitis and nephritis due to hypotensive injury. This complication was resolved with conservative management within 1 week. She had similar attack of cyanosis and worsening saturation and chest infection on postpartum day 10 which was managed with morphine, injectable antibiotics, and intubation. She became asymptomatic thereafter and was discharged in a stable condition after 3 weeks with proper counseling and advice for contraception and regular follow-up.

\section{Discussion}

Uncorrected TOF with pulmonary atresia in pregnancy is a rare entity with limited literature. The incidence of TOF with pulmonary atresia is $0.007 / 1000$ live births and accounts for one-fifth of all cases of TOF [1]. Although survival into adulthood without correction is rarely reported, but development of congenital systemic-pulmonary collaterals may enable patients with pulmonary atresia to reach adult age as in our patient. Management of these patients in pregnancy is challenging because of associated hemodynamic alterations. Increase in the plasma volume and the concomitant fall in both pulmonary vascular resistance and the systemic arterial resistance increase the right-to-left shunt during pregnancy, which further worsens hypoxia and cyanosis. Chronic hypoxia triggers secondary polycythemia which in turn depresses thrombopoeisis. 
Hypoxia and polycythemia both are risk factors for respiratory tract infection which further worsens dyspnea. Antithrombotic therapy is controversial and has to be balanced with increased risk of thrombosis due to polycythemia versus increased bleeding risk. As with hematocrit above $65 \%$, pregnancy wastage is virtually $100 \%$ [3] we decided to terminate pregnancy in our patient at $63 \%$ hematocrit. The polycythemia gradually improved after delivery.

Maternal complications in these women include worsening cyanosis and dyspnea, right ventricular failure, thromboembolism, and maternal mortality. Two case series, comprising 14 patients of TOF with pulmonary atresia with no corrective surgery, report a total of 35 pregnancies with successful pregnancy outcomes in 10 cases with live birth rates of 51 and $44 \%$, respectively $[4,5]$. Presbitero concluded that the probability of live birth could be predicted by hemoglobin of $>20 \mathrm{gm} \%$ and saturation $<85 \%$. In all of these studies, the percentage of live births was lower in patients with cyanosis than in acyanotic patients. To reduce these problems, sufficient rest and oxygen therapy have been suggested. Maternal mortality with pulmonary atresia is reported to be low with only two maternal deaths reported $[5,6]$. There is lack of evidence regarding the optimal time and mode of delivery in these patients. Careful postpartum monitoring is essential in these patients as sudden cardiovascular alterations in the postpartum period may be deterimental in these patients. Active management of third stage of labor and prevention of postpartum hemorrhage are mandatory as even lesser blood loss can lead to decompensation in these women as in our case.

Fetal complications include abortions, intrauterine growth restriction, prematurity, and intrauterine demise. Maternal cyanosis and polycythemia are the main threats leading to fetal growth restriction especially with oxygen saturation below $85 \%$. Multidisciplinary approach and strict vigilance are necessary for favorable maternal and fetal outcomes.

Compliance with ethical requirements and Conflict of interest Ethical approval and consent of patient was obtained prior to writing the case report. The authors report no conflict of interests.

\section{References}

1. Hofman JI. Incidence of congenital heart disease: I. Postnatal incidence. Pediatr Cardiol. 1995;16:103-13.

2. Veldtman GR, Connolly HM, Grogan M, et al. Outcomes of pregnancy in women with tetralogy of Fallot. J Am Coll Cardiol. 2004;44(1):174-80.

3. Warnes CA. Cyanotic congenital heart disease. In: Oakley C, Warnes CA, editors. Heart disease in pregnancy. 2nd ed. Massachusetts: Blackwell Publishing; 2007. p. 43-7.

4. Connolly HM, Warnes CA. Outcome of pregnancy in patients with complex pulmonic valve atresia. Am J Cardiol. 1997;79(4):519-21.

5. Neumayer U, Somerville J. Outcome of pregnancies in patients with complex pulmonary atresia. Heart. 1997;78(1):16-21.

6. Presbitero P, Somerville J, Stone S, et al. Pregnancy in cyanotic congenital heart disease. Outcome of mother and fetus. Circulation. 1994;89(6):2673-2676. 